\title{
Exogenous GABA Stimulates Endogenous GABA and Phenolic Acid Contents in Tomato Plants under Salt Stress
}

\author{
Fazilet Özlem Çekiç \\ Department of Biology, Faculty of Science and Letters, Aksaray University, 68100, Aksaray, TURKEY \\ Tel.:+903822882188 \\ ozlemcekic@aksaray.edu.tr \\ Received: 3 November 2017 \\ Accepted: 13 March 2018 \\ DOI: $10.18466 /$ cbayarfbe. 348935
}

\begin{abstract}
Gamma aminobutyric acid (GABA) is a non protein amino acid found in various organisms including plants. In recent years, the signal role of GABA in the stress response has of special interest. However, the effects of exogenous GABA on phenolic compounds which have special roles as antioxidants are not well known. In this study, the effects of exogenous GABA on endogenous GABA and phenolic contents were analyzed in tomato plants under salt stress. Tomato plants were grown in a growth chamber under controlled conditions and $\mathrm{NaCl}$ and $\mathrm{GABA}$ were applied in Hoagland solution. Qualitative and quantitative analysis of GABA and phenolic compounds (Benzoic, caffeic, chlorogenic, gallic, hydroxybenzoic, syringic, rosmarinic, pcoumaric, sinapic, t-cinnamic, t-ferulic acids, catechin, epicatechin, hesperidin and quercetin) were measured by HPLC. Differences were found in chlorogenic acid, coumaric acid and gallic acid among the phenolic substances. We found a significant increase in gallic acid and coumaric acid contents under $200 \mathrm{mM} \mathrm{NaCl}$ and GABA applications. Exogenous GABA treatment caused a slight increase in endogenous GABA content. The increase in GABA content under GABA+salt treatments were higher than that of single salt and GABA applications. According to our results we can suggest that exogenous GABA could enhance the stress response by enhancing some phenolic substances and GABA content under salt stress.
\end{abstract}

Keywords: Gamma-aminobutyric acid, HPLC, Salinity, Solanum lycopersicon.

\section{Introduction}

Gamma aminobutyric acid (GABA) is an important non protein amino acid that is found in various organisms including plants. In animals, GABA has an important role as an inhibitor neurotransmitter. Moreover, in plants it has special impacts on plant metabolism such as $\mathrm{pH}$ regulation, and $\mathrm{C}-\mathrm{N}$ balance [1-3].

In the GABA shunt, $\alpha$-ketoglutarate is converted to glutamate by the activity of glutamate dehydrogenase (GDH, EC 1.4.1.4). Then GABA is occurred from glutamate by the activity of glutamate decarboxylase (GAD, EC 4.1.1.15). In addition, GABA can be synthesized via polyamine degradation. Abiotic and biotic stresses can cause cytosolic acidification and low $\mathrm{pH}$ activates glutamate decarboxylase and GABA can be accumulated rapidly in various parts of plants [3]. Also environmental stress factors can increase cytosolic $\mathrm{Ca}^{2+}$ which activates calmodulin-dependent glutamate decarboxylase and GABA synthesis $[1,3]$. Moreover, in recent years GABA is defined as a signal molecule $[4,5]$.
In previous studies, it has been reported that exogenous application of GABA could alleviate the deleterious effects of stress conditions. Exogenous GABA could stimulate polyamine biosynthesis and degradation under stress [6]. It was also reported that GABA could participate in regulating of gene expression as a signal molecule under stress conditions [7].

Phenolic compounds have also important roles in the defense mechanism of higher plants. Their biosynthesis is often enhanced by various environmental stresses, such as salinity. Moreover, they can act as potential antioxidants by eliminating the deleterious effects of ROS induced by salt stress [8]. However, the impact of exogenous GABA treatments on phenolic compounds in tomato plants under stress conditions is not well known. In this study, our aim was to determine the effects of exogenous GABA on the contents of phenolic substances and GABA in tomato plants under salt stress.

\section{Materials and Methods}

\subsection{Growth conditions and stress treatment}

The seeds of tomato plants Solanum lycopersicum L. cv. H-2274 were provided from Anatolia Agricultural 
Research Institute in Eskisehir, Turkey. First, the seeds were surface sterilized in $5 \% \mathrm{NaOCl}$ and washed thoroughly with sterile water and then germinated on perlite in a growth chamber under $26^{\circ} \mathrm{C} / 22^{\circ} \mathrm{C}, 168 \mathrm{~h}$ light/dark, with a relative humidity of $65 \%$ and a light intensity of $175 \mu \mathrm{mol} \mathrm{m} \mathrm{m}^{-2} \mathrm{~s}^{-1}$. The seedlings were watered regularly with $1 / 2$ Hoagland solution (Hoagland, Sigma H2395). After 4 weeks 0, 100, $200 \mathrm{mM} \mathrm{NaCl}$ and/or $0.5 \mathrm{mM}$ GABA were applied in Hoagland solution for one week. At the harvest, the leaves were frozen in liquid nitrogen and stored at $-80{ }^{\circ} \mathrm{C}$ until analyses.

\subsection{GABA analysis}

GABA analysis was performed with HPLC (Agilent 1200). $0.2 \mathrm{~g}$ of leaf samples were homogenized in $5 \mathrm{~mL}$ solution of water:chloroform:methanol (3:5:12). The samples were then centrifuged for $5 \mathrm{~min}$ at $10,000 \mathrm{~g}$ at 4 ${ }^{\circ} \mathrm{C}$, and the supernatant was dried. In order to derivatizate with 2-hydroxynaphthaldehyde (HN), the samples were dissolved in $100 \mu \mathrm{L}$ of ultrapure water and $150 \mu \mathrm{L}$ of Borax buffer ( $\mathrm{pH} 8$ ) and $250 \mu \mathrm{L}$ of $\mathrm{HN}$ $(0.3 \%)$. The mixture was kept at $80{ }^{\circ} \mathrm{C}$ for $30 \mathrm{~min}$ and then cooled at room temperature. $1 \mathrm{~mL}$ of methanol was added to the samples. Samples were separated by reversed-phase column Supelco LC18 (250x4.6 mm, 5 $\mu \mathrm{m})$ with the injection volume of $5 \mu \mathrm{L}$, flow rate of 1 $\mathrm{mL} \min ^{-1}$, and mobile phase of methanol:water (62:38). The analysis was carried out at $330 \mathrm{~nm}$ wavelength. The retention time was $12 \mathrm{~min}$. The peak areas were compared with the GABA standards and calculated for determining of the GABA content [9].

\subsection{Phenolic compound analysis}

Qualitative and quantitative analyses of phenolic compounds were analyzed by HPLC (Agilent 1200). 0.1 $\mathrm{g}$ of leaf samples were homogenized in methanol (HPLC grade), and centrifuged at $10,000 \mathrm{~g}$ for $10 \mathrm{~min}$. The samples were filtered with $0.45 \mu \mathrm{m}$ filters and separated by reversed-phase column Supelco LC18 $(250 \times 4.6 \mathrm{~mm} 2,5 \mu \mathrm{m})$ with an injection volume of 20 $\mu \mathrm{L}$ and flow rate of $0.8 \mathrm{~mL} \mathrm{~min} \mathrm{~m}^{-1}$ at $278 \mathrm{~nm}$. $2 \%$ of acetic acid and methanol were used as mobile phase and applied gradiently as described by Caponio et al. [10]. Each sample was analyzed for $90 \mathrm{~min}$. Quantifications were done by comparing the peak areas with phenolic compounds standards (Benzoic, caffeic, chlorogenic, gallic, hydroxybenzoic, syringic, rosmarinic, pcoumaric, sinapic, t-cinnamic, t-ferulic acids, catechin, epicatechin, hesperidin and quercetin).

\subsection{Statistical analysis}

The effects of exogenous GABA application were determined using one-way variance analysis ANOVA. The applications were compared by least significant difference (LSD) test at $\mathrm{p}<0.05$. The spread of values was shown in the figures as standard errors of the means.

\section{Results and Discussion}

Gamma aminobutyric acid is an important metabolite that has essential roles in the primary metabolism of plants $[4,11]$. In recent years, GABA is mentioned as a signal molecule under various stress conditions. Accumulation of GABA in stressed tissues may activate the regulation of the expression of genes that have roles in the detoxification of ROS [7,12]. Therefore, GABA could help to maintain the cells from oxidative damage and enhance the stress response in plants [13]

Recent studies have mentioned that endogenous GABA level can be increased by exogenous GABA application depending on the GABA concentration [14,15]. In a previous study, GABA treatment caused a remarkable increase in endogenous GABA concentration in the roots of melon plants under both normal and hypoxia stress conditions. The highest increase was reported in stress+GABA application. We are in agreement with these findings with an increase in endogenous GABA concentration by GABA treatment under both stressed and non-stressed conditions (Figure 1). This increase could help to induce defense mechanism against stress conditions in tomato plants.

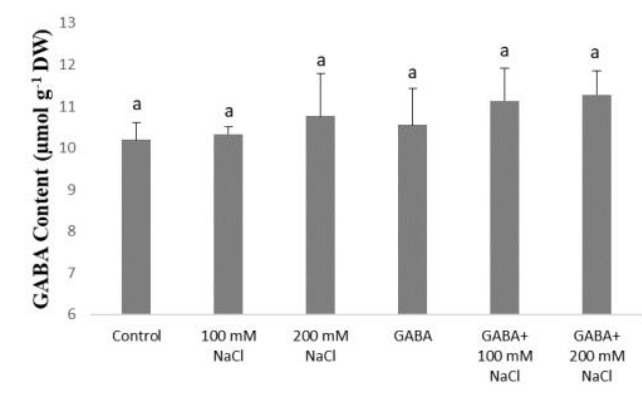

Figure 1. The effects of exogenous GABA application and salt stress on endogenous GABA content in the leaves of $S$. lycopersicon. The bars represent the means \pm S.E $(\mathrm{p}<0.05)$.

Parallel to our study, endogenous GABA level was induced by $\mathrm{NaCl}$ stress, however the application of GABA under salt stress increased endogenous GABA concentration more than single salt treatment [7]. Wang et al. [6] also reported that the increase in GABA concentration could help to inhibit the deleterious effects of stress by maintaining cellular $\mathrm{pH}$ and promoting of the TCA cycle. In melon plants, GABA application was mentioned to eliminate the deleterious effects of hypoxia stress by enhancing the polyamine biosynthesis and prevents the degradation of polyamines which have important roles under stress conditions [6]. Alqarawi et al. [16] reported that GABA treatment increased antioxidant enzymes activities and could protect the cells against the deleterious effects of salt stress by maintaining of the hormones and mineral nutrients and by reducing lipid peroxidation. Another important impact of GABA was its preventing role in 
the increase in sodium and chloride ion levels under salt stress.

In addition, in the postharvest process GABA application can have positive effects on preserving the quality of the fruits. Sheng et al. [15] reported that exogenous GABA could prevent the decrease of organic acids during postharvest storage and suggested GABA treatment for enhancing the postharvest quality. GABA is also suggested as an important supplement for increasing of the fruit resistance against adverse conditions. In addition, GABA could help to increase the flavor and the quality of the fruits during postharvest. Therefore, the effects of exogenous GABA on plant physiology should be well evaluated for enhancing crop yield and quality.

In our study, we also determined the effects of GABA on phenolic substances in tomato plants under salt stress. Qualitative and quantitative analyses of 15 phenolic compounds (Benzoic, caffeic, chlorogenic, gallic, hydroxybenzoic, syringic, rosmarinic, pcoumaric, sinapic, t-cinnamic, t-ferulic acids, catechin, epicatechin, hesperidin and quercetin) were measured by HPLC. Under these conditions we found differences in chlorogenic acid, coumaric acid and gallic acid among the phenolic compounds (Figure 2, 3, 4).

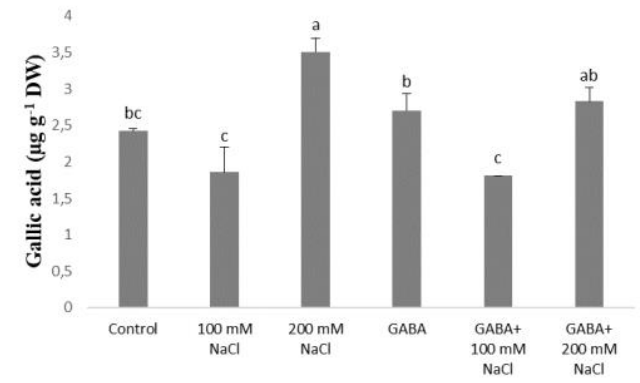

Figure 2. The effects of exogenous GABA application and salt stress on gallic acid content in the leaves of $S$. lycopersicon. The bars represent the means \pm S.E. Means with different letters are significantly different ( $\mathrm{p}$ $<0.05)$.

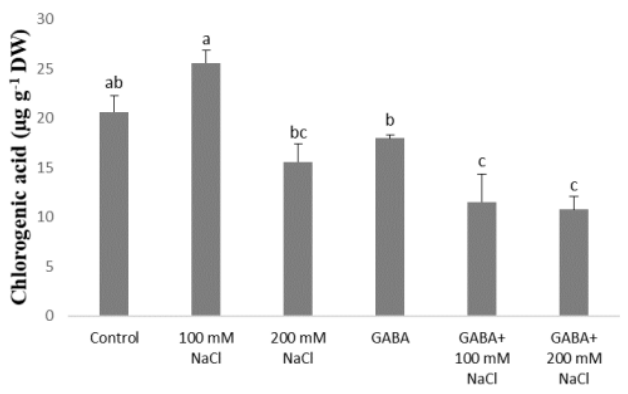

Figure 3. The effects of exogenous GABA application and salt stress on chlorogenic acid content in the leaves of $S$. lycopersicon. The bars represent the means \pm S.E. Means with different letters are significantly different ( $p$ $<0.05)$.

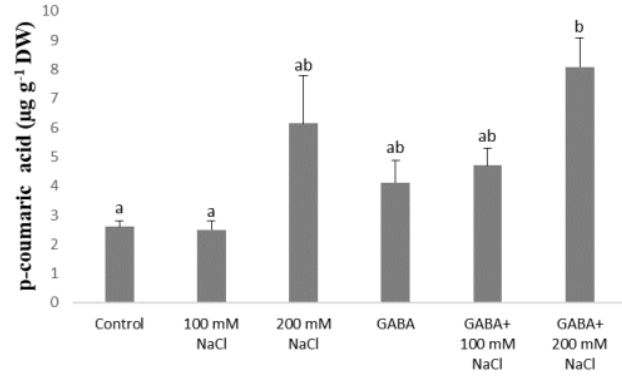

Figure 4. The effects of exogenous GABA application and salt stress on p-coumaric acid content in the leaves of $S$. lycopersicon. The bars represent the means \pm S.E. Means with different letters are significantly different ( $\mathrm{p}$ $<0.05)$.

The roles of phenolic acids as antioxidants have been mentioned because of their properties related to the $\mathrm{H}$ donating ability of the phenols. Therefore, the enhancement in the phenolic substances can help to scavenge the reactive oxygen species [17]. Gallic acid, chlorogenic acid and coumaric acid are known to have the ability of radical scavenging. We found a significant increase in gallic acid and coumaric acid contents under $200 \mathrm{mM} \mathrm{NaCl}$ application when compared to control plants. Gallic acid and coumaric acid contents were also enhanced by GABA application. However, chlorogenic acid content was decreased by GABA and GABA+ salt applications as compared to control plants. $100 \mathrm{mM}$ and $200 \mathrm{mM} \mathrm{NaCl}$ applications caused an increase in GABA content as compared to control plants. We can suggest that exogenous GABA could enhance the stress response by enhancing gallic acid and coumaric acid contents under salinity (Figure 2,4).

\section{Conclusion}

According to our results, we can conclude that exogenous GABA application can enhance some phenolic substances and GABA content under salt stress. The increase in these substances could help to enhance defense strategies in tomato plants under salinity. However, further studies should be done to understand the role of exogenous GABA on the signal and defense mechanisms under stress conditions.

\section{References}

1. Kinnersley, AM, Turano, FJ, Gamma aminobutyric acid (GABA) and plant responses to stress, Critical Reviews in Plant Sciences, 2000, 479-509.

2. Bouche, N, Fromm, H, GABA in plants:just a metabolite? Trends in Plant Science, 2004, 9(3), 110-115.

3. Shelp, BJ, Mullen, RT, Waller, JC, Compartmentation of GABA metabolism raises intriguing questions. Trends in Plant Science, 2012, 17(2), 57-9.

4. Michaeli, S, Fromm, H, Closing the loop on the GABA shunt in plants: Are GABA metabolism and signaling entwined? Frontiers in Plant Science, 2015, 6:1-7. 
5. Ramesh, SA, Tyerman, SD, Gilliham, M, Xu, B, $\gamma$-Aminobutyric acid (GABA) signalling in plants, Cellular and Molecular Life Sciences, 2017, 74, 1577-1603.

6. Wang, C, Fan, L, Gao, H, Polyamine biosynthesis and degradation are modulated by exogenous gamma-aminobutyric acid in root-zone hypoxia-stressed melon roots, Plant Physiology Biochemistry, 2014, 82:17-26.

7. Shi, SQ, Shi, Z, Jiang, ZP, Effects of exogenous GABA on gene expression of Caragana intermedia roots under $\mathrm{NaCl}$ stress: regulatory roles for $\mathrm{H}_{2} \mathrm{O}_{2}$ and ethylene production, Plant Cell Environment, 2010, 33:149-62.

8. Petridis, A, Therios, I, Samouris, G, Tananaki, C, Salinityinduced changes in phenolic compounds in leaves and roots of four olive cultivars (Olea europaea L.) and their relationship to antioxidant activity, Environmental and Experimental Botany, 2012, 79, 37-43.

9. Bor, M, Seckin, B, Ozgur, R, Yılmaz, O, Ozdemir, F, Turkan, I Comparative effects of drought, salt, heavy metal and heat stresses on gamma-aminobutryric acid levels of sesame (Sesamum indicum L.). Acta Physiologiae Plantarum, 2009, 31(3), 655-659.

10. Caponio, F, Alloggio, V, Gomes, T, Phenolic compounds of virgin olive oil: Influence of paste preparation techniques. Food Chemistry, 1999, 64(2), 203-209.

11. Deleu, C, Faes, P, Niogret, MF, Bouchereau, A, Effects of the inhibitor of the $\gamma$-aminobutyrate-transaminase, vinyl- $\gamma$ aminobutyrate, on development and nitrogen metabolism in Brassica napus seedlings, Plant Physiology and Biochemistry, 2013, 64:60-9.

12. Paradisone, $\mathrm{V}$, Barrameda-Medina, $Y$, Montesinos-Pereira, $D$, $m$ Romero, L, Esposito, S, Ruiz, JM, Roles of some nitrogenous compounds protectors in the resistance to zinc toxicity in Lactuca sativa cv. Phillipus and Brassica oleracea cv. Bronco. Acta Physiologia Plantarum, 2015, 37:1-8.

13. Kinnersley, AM, Lin, F, Receptor modifiers indicate that 4 aminobutyric acid (GABA) is a potential modulator of ion 20 transport in plants. Plant Growth Regulation, 2000, 32:65-76.

14. Li, Y, Fan, Y, Ma, Y, Zhang, Z, Yue, H, Wang, L, Jiao, Y, Effects of exogenous $\gamma$-aminobutyric acid (GABA) on photosynthesis and antioxidant system in Pepper (Capsicum annuum L.) seedlings under low light stress. Journal of Plant Growth Regulation, 2017, 36(2), 436-449.

15. Sheng, L, Shen, D, Luo, Y, Exogenous $\gamma$-aminobutyric acid treatment affects citrate and amino acid accumulation to improve fruit quality and storage performance of postharvest Citrus fruit. Food Chemistry, 2017, 216:138-145.
16. Alqarawi, AA, Hashem, A, Abd-Allah, EF, Al-Huqail, AA, Alshahrani, TS, Alshalawi, SR, Egamberdieva, D, Protective role of gamma amminobutyric acid on Cassia italica Mill. under salt stress, Legume Research, 2016, 39, 396-404.

17. Rice-Evans, NCA, GeorgePaganga JMG, Structure-antioxidant activity relationships of flavonoids and phenolic acids, Free Radical Biology and Medicine, 1996, 20 (7) 933-956.

\section{Supplementary materials}

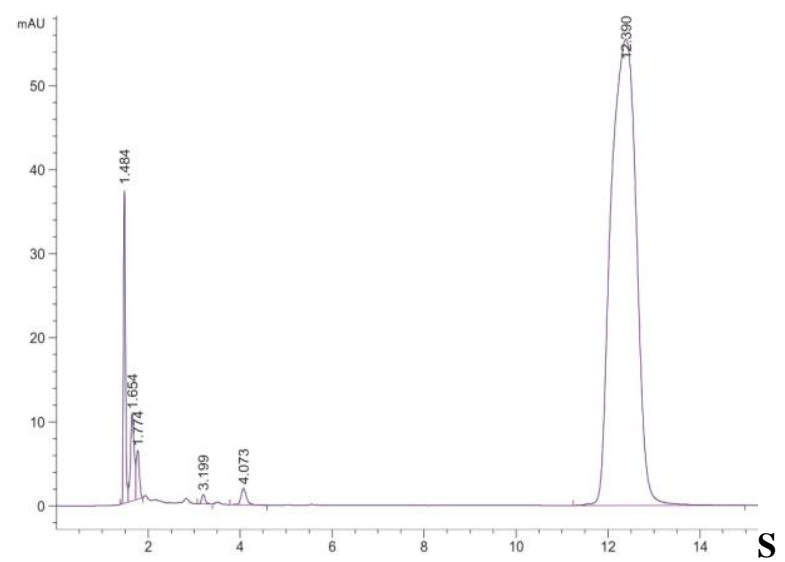

P 1. HPLC chromatogram of GABA. The retention time was $12 \mathrm{~min}$.

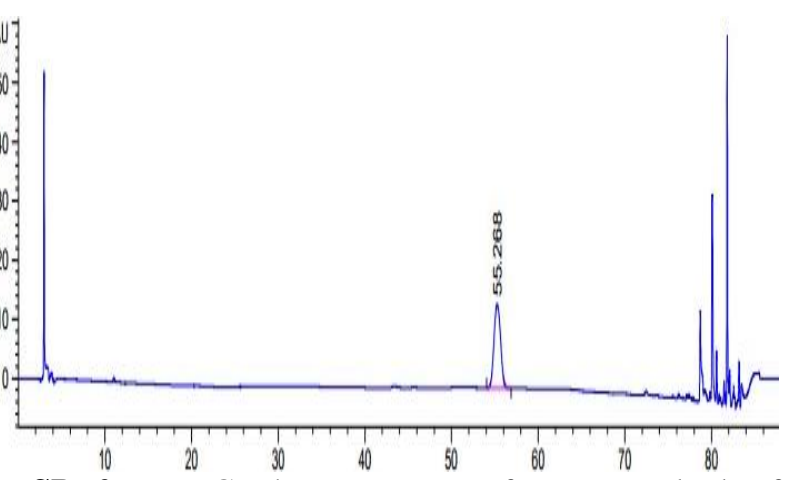

SP 2. HPLC chromatogram of one standard of a phenolic compound. Each sample was analyzed for 90 $\min$. 